Article

\title{
Experimental Interference Robustness Evaluation of IEEE 802.15.4-2015 OQPSK-DSSS and SUN-OFDM Physical Layers for Industrial Communications
}

\author{
Pere Tuset-Peiró ${ }^{1, *(\mathbb{D}}$, Francisco Vázquez-Gallego ${ }^{2}\left(\mathbb{D}\right.$, Jonathan Muñoz ${ }^{3}$, Thomas Watteyne ${ }^{3}(\mathbb{D}$, \\ Jesus Alonso-Zarate ${ }^{2} \mathbb{1}$ and Xavier Vilajosana ${ }^{1}$ (1) \\ 1 Wireless Networks Research Laboratory, Internet Interdisciplinary Institute (IN3), Universitat Oberta de \\ Catalunya (UOC), 08060 Castelldefels, Barcelona, Spain; xvilajosana@uoc.edu \\ 2 M2M Department, Centre Tecnològic de Telecomunicacions de Catalunya (CTTC), 08060 Castelldefels, \\ Barcelona, Spain; fvazquez@cttc.es (F.V.-G.); jesus.alonso@cttc.es (J.A.-Z.) \\ 3 EVA Team, Institut National de Recherche en Informatique et en Automatique (INRIA), 75012 Paris, France; \\ jonathan.munoz@inria.fr (J.M.); thomas.watteyne@inria.fr (T.W.) \\ * Correspondence: peretuset@uoc.edu
}

Received: 19 August 2019; Accepted: 13 September 2019; Published: 18 September 2019

\begin{abstract}
In this paper, we experimentally evaluate and compare the robustness against interference of the OQPSK-DSSS (Offset Quadrature Phase Shift Keying-Direct Sequence Spread Spectrum) and the SUN-OFDM (Smart Utility Network-Orthogonal Frequency Division Multiplexing) physical layers, as defined in the IEEE 802.15.4-2015 standard. The objective of this study is to provide a comprehensive analysis of the impact that different levels of interference produce on these modulations, in terms of the resulting PDR (Packet Delivery Ratio) and depending on the length of the packet being transmitted. The results show that the SUN-OFDM physical layer provides significant benefits compared to the ubiquitous OQPSK-DSSS in terms of interference robustness, regardless of the interference type and the packet length. Overall, this demonstrates the suitability of choosing the SUN-OFDM physical layer when deploying low-power wireless networks in industrial scenarios, especially taking into consideration the possibility of trading-off robustness and spectrum efficiency depending on the application requirements.
\end{abstract}

Keywords: IEEE 802.15.4; smart utility networks; OQPSK-DSSS; SUN-OFDM; interference

\section{Introduction}

The IEEE 802.15.4 standard [1] was first released in May 2003 and defined a physical layer (PHY) and a MAC (Medium Access Control) layer for WPAN (Wireless Personal Area Networks) operating in the sub-GHz (868 MHz in Europe, $915 \mathrm{MHz}$ in America) and the $2.4 \mathrm{GHz}$ worldwide ISM (Industrial, Scientific and Medical) frequency bands [2]. The PHY layer was built upon the DSSS-OQPSK (Direct Sequence Spread Spectrum-Offset Quadrature Phase Shift Keying) modulation and provided data rates of $20 \mathrm{kbps}$ and $40 \mathrm{kbps}$ in the sub-GHz bands, respectively, and of $250 \mathrm{kbps}$ in the $2.4 \mathrm{GHz}$ band. At the MAC layer, the standard defined slotted/synchronized and unslotted/unsynchronized operation based on the CSMA/CA (Carrier Sense Multiple Access with Collision Avoidance) channel access mechanism to trade-off bandwidth, latency and energy consumption of the devices.

Thanks to its simplicity and low-cost, over the years, the IEEE 802.15.4 standard has become the basis for multiple low-power wireless communications technologies including ZigBee [3], ISA100.11a [4], WirelessHART [5] and 6TiSCH [6], among others. In fact, the adoption of the IEEE 802.15.4 standard by different standardization bodies and technologies has promoted the revision of the standard (i.e., three times: in 2006, 2011 and 2015) in order to clarify the operation and to add 
new features to both the PHY and MAC layers. For example, the 2015 standard revision adopted the MAC layer protocols defined in the IEEE 802.15.4e-2012 [7] amendment. Among others, this amendment defined the TSCH (Time Slotted Channel Hopping), a channel access mechanism that combines TDMA (Time Division Multiple Access) and FDMA (Frequency Division Multiple Access) to support industrial requirements, including reliable packet delivery (i.e., 99.999\%) in adverse conditions such as multi-path propagation and external interference.

Similarly, the IEEE 802.15.4-2015 standard revision included three new physical layers targeted to SUN (Smart Utility Network) applications, SUN-FSK, SUN-OQPSK and SUN-OFDM, as defined in the IEEE 802.15.4g-2012 [8] amendment. The SUN-FSK and SUN-OQPSK modulations focus on maintaining backwards compatibility with previous standards and commercially available transceivers, whereas the SUN-OFDM focuses on adding robustness and improving spectrum efficiency at the physical layer. The benefits brought by SUN-OFDM are mainly due to the use of parallel transmissions with orthogonal sub-carriers, each transporting a small portion of the information to be transmitted using a narrow-band modulation. Such approach provides better robustness against multi-path propagation (as sub-channels can be considered flat fading, which are inherently robust to inter-symbol and inter-frame interference) and external interference (as narrow-band interference only affects a portion of the sub-channels and frequency repetition can be used to overcome its effects), as well as improving spectrum efficiency (sub-channels are optimally spaced, allowing to trade-off robustness or data-rate depending on the application requirements).

The consolidation of the IEEE 802.15.4g-2012 amendment into the IEEE 802.15.4-2015 standard, as well as the appearance of the first commercially available radio transceivers supporting it, entails a shift in the design, development and deployment of low-power wireless networks, with special interest in the industrial domain, where the propagation and interference effects have largely slowed or limited the adoption of such technologies. Taking that into account, in this paper, we focus on empirically evaluating the interference robustness of the SUN-OFDM and compare it against OQPSK-DSSS. Our goal is to provide evidence of the robustness of the different modulations, including their possible configurations and under different operating conditions, to understand the benefits and limitations when deploying such technologies. To the best of our knowledge, these results are novel and help researchers and practitioners deploy low-power wireless networks that are more robust and make an efficient use of the spectrum in real-world scenarios.

The remainder of this article is organized as follows. Section 2 provides an overview on the PHYs defined in the IEEE 802.15.4-2015 standard that are evaluated in this article. Section 3 provides a survey of the research related to the SUN-OFDM physical layer for low-power wireless networks. Section 4 presents the methodology and the setup used to conduct the experiments to evaluate the robustness of the SUN-OFDM and OQPSK-DSSS PHYs. Section 5 presents and summarizes the results obtained from the measurement campaign. Section 6 discusses the results obtained and presents a series of recommendations. Finally, Section 7 concludes this article.

\section{IEEE 802.15.4-2015 Overview}

The 2015 version of the IEEE 802.15.4 standard offers 18 different physical layers, each targeting specific applications and market segments. In this section, we present and briefly describe the two physical layers analyzed in this paper, i.e., OQPSK-DSSS and SUN-OFDM.

\subsection{IEEE 802.15.4-2015 OQPSK-DSSS}

OQPSK-DSSS was introduced in the original IEEE 802.15.4 standard in 2003 and it uses a 16-ary quasi-orthogonal modulation where each data symbol represents 4 bits of information. Therefore, there are 16 possible symbols and each of them is represented by a PRNG (Pseudo-Random Noise Generator) chip sequence that is almost orthogonal.

When operating in the $780 \mathrm{MHz}, 915 \mathrm{MHz}, 2380 \mathrm{MHz}$ and $2450 \mathrm{MHz}$ bands, the PRN sequence is 32 chips long for 4 bits of data, hence providing a data rate of $250 \mathrm{kbps}$. In contrast, when operating in 
the $868 \mathrm{MHz}$ band, the PRN sequence is 16 chips long for 4 bits of data, thus offering a data rate of 100 kbps.

As depicted in Figure 1, OQPSK-DSSS frames are composed of an SHR (Synchronization Header), PHR (PHY Header) and PSDU (PHY Service Data Unit). The SHR contains a preamble and an SFD (Start of Frame Delimiter); the PHR contains the frame length (7 bits) and a reserved bit. Hence, the maximum length of the PSDU is 127 bytes.

\begin{tabular}{|c|c|c|cc|}
\hline \multicolumn{2}{|c|}{ SHR } & PHR & PHY Payload \\
\hline $\begin{array}{c}\text { Preamble } \\
\text { (4 octets) }\end{array}$ & $\begin{array}{c}\text { SFD } \\
\text { (1 octet) }\end{array}$ & $\begin{array}{c}\text { PHY Header } \\
\text { (1 octet) }\end{array}$ & $\begin{array}{c}\text { PSDU } \\
\text { (variable, } \leq 127 \text { bytes) }\end{array}$ \\
\hline \\
$\qquad \begin{array}{c}\text { Frame length } \\
\text { (7 bits) }\end{array}$ & $\begin{array}{c}\text { Reserved } \\
\text { (1 bits) }\end{array}$ \\
\hline
\end{tabular}

Figure 1. IEEE 802.15.4-2015 OQPSK-DSSS frame format.

\subsection{IEEE 802.15.4 SUN-OFDM}

The SUN-OFDM (Orthogonal Frequency Division Multiplexing) physical layer was rolled into the IEEE 802.15.4-2015 version of the standard along with SUN-FSK (Frequency Shift Keying) and SUN-OQPSK (Offset Quadrature Phase Shift Keying). While FSK and OQPSK are two well-known modulation techniques that have been widely used in low-power wireless communications thanks to their simplicity, performance and low-cost, OFDM has been commonly used in other communication domains such as fixed and cellular access (e.g., xDSL, WiMAX and LTE), as well as wired and wireless LANs (e.g., PLC and Wi-Fi) due to the stringent processing, memory and energy consumption requirements.

The SUN-OFDM PHY can operate in both the sub-GHz bands (400, 700, 800 and $900 \mathrm{MHz})$ and the $2.4 \mathrm{GHz}(2400-2483.5 \mathrm{MHz})$ with data rates from $50 \mathrm{kbps}$ to $800 \mathrm{kbps}$ depending on the OFDM option and MCS (Modulation and Coding Scheme) value, as summarized in Table 1.

As it can be observed in Table 1, SUN-OFDM defines four options, numbered from 1 to 4 , which determine how many sub-carriers are grouped together in order to form an OFDM channel. Option 1 , the largest, groups 104 sub-carriers and occupies a bandwidth of $1094 \mathrm{kHz}$ with $1200 \mathrm{kHz}$ of channel spacing. Option 4, the narrowest, groups 14 sub-carriers and occupies a bandwidth of $156 \mathrm{kHz}$ with $200 \mathrm{kHz}$ of channel spacing.

On top of that, the MCS parameter, numbered from 0 to 6 , determines how each sub-carrier is modulated (available modulation for each carrier are BPSK, QPSK and 16-QAM), whether frequency repetition is applied (i.e., $4 \times, 2 \times$ or no frequency repetition) and the FEC (Forward Error Correction) rate applied to the input data stream for each sub-carrier (i.e., $R=1 / 2$ or $R=3 / 4$ ).

Regardless of the OFDM option and MCS value, the sub-carrier spacing and symbol rate is constant: $10416-2 / 3 \mathrm{~Hz}$ and 8-1/3 ksymbols/s. Each symbol in the OFDM physical layer is $120 \mu \mathrm{s} \mathrm{long,}$ although the actual symbol transmission time is divided into a BS (Base Symbol) time of $96 \mu$ s and a

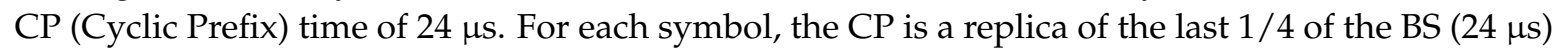
and is copied at the front, making the signal periodic. The long duration of an OFDM symbol and the fact that it is cyclic makes it robust against long spread time environments resulting in multi-path propagation that may cause ISI (Inter-Symbol Interference) [9].

As per the IEEE 802.15.4-2015 standard, SUN-OFDM frames are composed of a SHR (Synchronization Header), PHR (PHY Header) and PSDU (PHY Service Data Unit), as shown in Figure 2. The SHR is composed of a short and long preamble (STF and LTF, respectively), whereas the PHR contains a header with the receiver configuration for the PHY payload (modulation and data rate, frame length, scrambler configuration and a redundancy check). The OFDM header (SHR and PHR) is transmitted using the lowest supported MCS level for the OFDM option being used. That is, for OFDM1 and OFDM2, the header is transmitted using MCS0 (BPSK, $\mathrm{R}=1 / 2,4 \times$ repetition), 
for OFDM3 the header is transmitted using MCS1 (BPSK, $\mathrm{R}=1 / 2,2 \times$ repetition), and, for OFDM4, the header is transmitted using MCS2 (QPSK, $\mathrm{R}=1 / 2,2 \times$ repetition). Therefore, for any device implementing any of the OFDM options, implementation of the BPSK and QPSK is mandatory and support of 16-QAM is optional. Finally, the maximum length of the PSDU is 2047 bytes, large enough to transport a complete IPv6 packet without fragmentation.

Table 1. SUN-OFDM parameters.

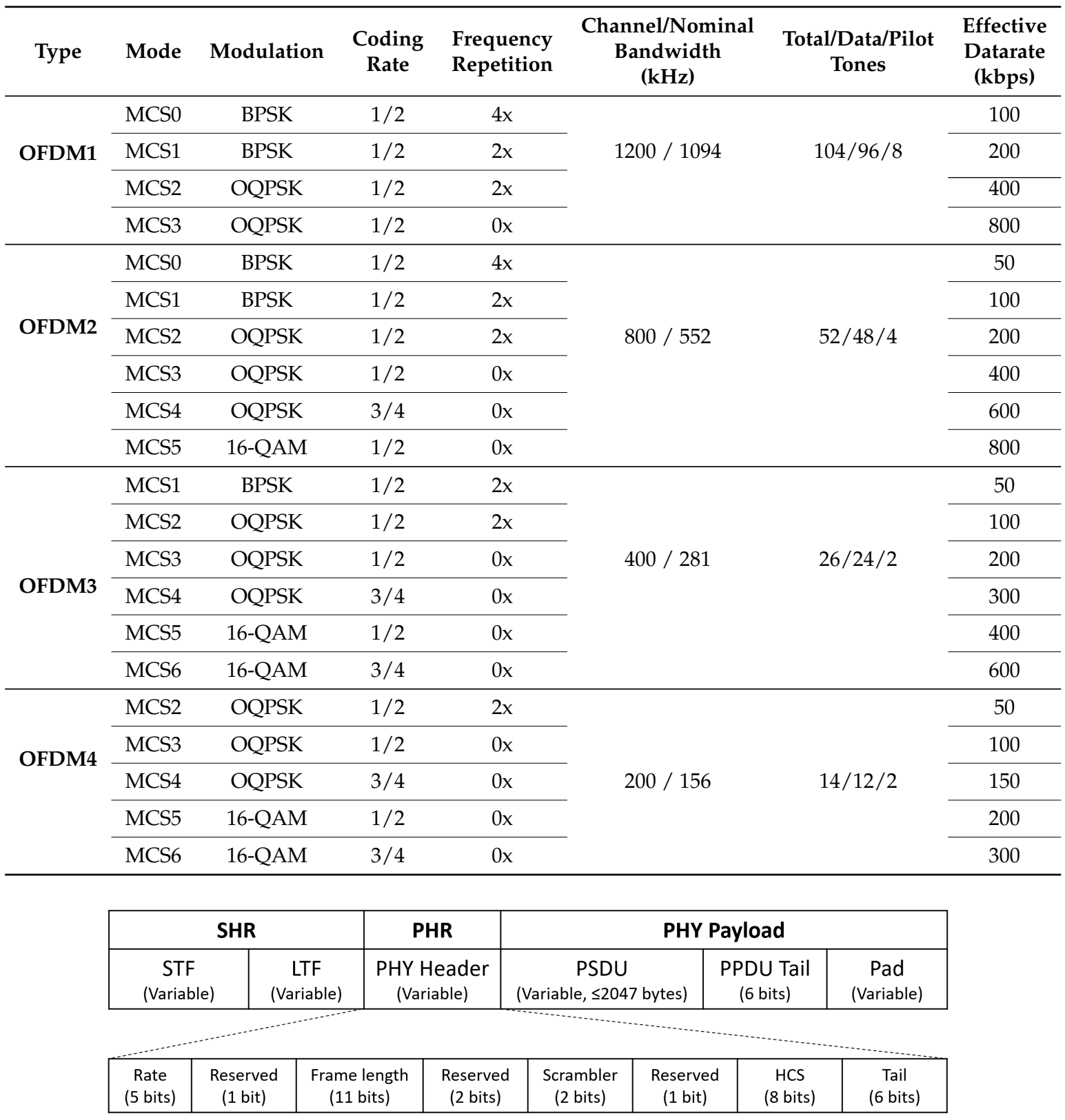

Figure 2. IEEE 802.15.4-2015 SUN-OFDM frame format.

\section{Related Work}

Despite being introduced in the IEEE 802.15.4g-2012 amendment, the SUN-OFDM PHY has not received a lot of attention from the research community to understand the potential benefits (and pitfalls) of applying this technology in low-power wireless communications for industrial applications. This is mainly due to the lack of commercially available radio transceivers that support all standard modes. In this section, we provide a summary of related work that has evaluated the 
performance of the PHYs proposed in the IEEE 802.15.4g-2012 amendment. The results are presented in chronological order.

In [10], the authors experimentally evaluate various LPWAN (Low-Power Wide-Area Network) technologies for industrial sensing applications, including condition monitoring. The results, which include LoRa (Long Range) and IEEE 802.15.4g (using SUN-FSK only), conclude that, with respect to IEEE 802.15.4g (SUN-FSK), LoRa provides the largest communication range $(2 \times)$ at the expense of an increased energy consumption $(10 \times)$.

In [11], the authors study the coexistence mechanisms of homogeneous and heterogeneous IEEE 802.15.4g systems for SUN, and perform a coexistence analysis to evaluate the performance degradation of a victim system in the presence of an interferer. They show that, with a distance over $30 \mathrm{~m}$, the victim and the interferer are able to coexist even without any higher layer mechanism that enforce coexistence.

In [12], the authors study the coexistence of IEEE 802.11 ah and IEEE 802.15.4 g networks, which provide communication ranges above $1 \mathrm{~km}$, and are specifically targeted at outdoor IoT (Internet of Things) applications. Simulation results show that co-located IEEE 802.11 ah networks can severely impact the operation of IEEE 802.15.4 g networks due to the higher ED (Energy Detection) threshold and the faster back-off mechanism. Moreover, the differences in modulation scheme and frame structure limit the capability to implement automatic mechanisms to enforce cooperation. Hence, the self-coexistence mechanism needs to be enforced to mitigate the effects of interference in the network performance.

More recently, in [13] and [14], the authors evaluate the suitability of the IEEE 802.15.4 g-2012 physical layers for environmental and smart building applications, respectively, showing that SUN-OFDM is affected by multi-path fading and external interference in a similar way as OQPSK-DSSS. Based on these results, in [15], the authors conclude that the SUN-OFDM physical layer alone does not yield the robustness required for industrial applications and, thus, they recommend to add channel hopping techniques to combat its effects.

\section{Methodology and Setup}

In this section, we present the research methodology that we have used to evaluate the interference robustness of the SUN-OFDM modes and compare it with the OQPSK-DSSS mode, both defined in the IEEE 802.15.4-2015 PHYs.

\subsection{Evaluation Methodology}

To evaluate the interference robustness of the SUN-OFDM PHY and compare it to the OQPSK-DSSS PHY, we use the setup depicted in Figure 3. The setup consists of a transmitter, a receiver and an interferer device connected through an RF (RadioFrequency) power combiner (further described in Section 4.3).

For the SUN-OFDM modulation, we select the operating modes that provide a similar data-rate of the OQPSK-DSSS mode, $250 \mathrm{kbps}$. In particular, we select the OFDM1-MCS1, OFDM2-MCS2, OFDM3-MCS3 and OFDM4-MCS5 modes that provide a data-rate of $200 \mathrm{kbps}$ but with different PHY properties (i.e., channel bandwidth, modulation, coding rate and frequency repetition) as presented in Section 2 and summarized in Table 2. 


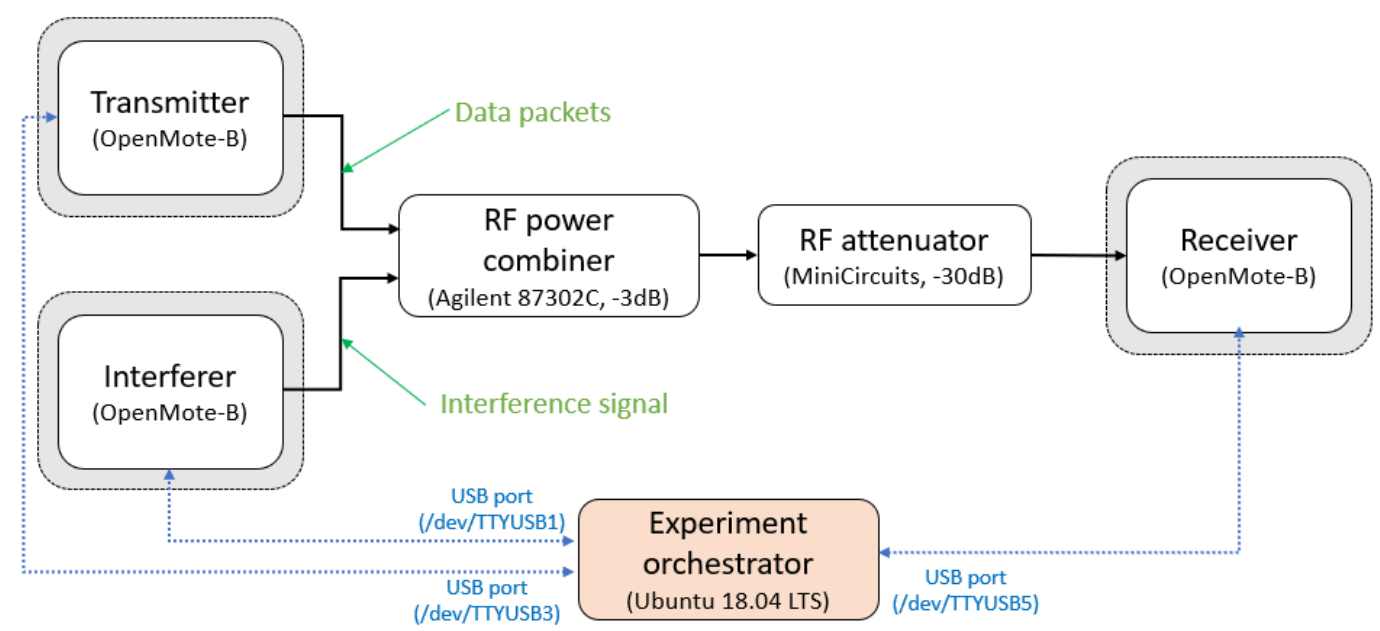

Figure 3. Evaluation setup. Note that the grey box around the transmitter, interferer and receiver devices represents an RF shielding enclosure used to ensure that communication between devices can only occur through the RF coaxial cables.

Table 2. IEEE 802.15.4-2015 modulations that have been selected in this study to evaluate their robustness against interference.

\begin{tabular}{ccccccccc}
\hline Name & Mode & Modulation & $\begin{array}{c}\text { Channel } \\
\text { Coding }\end{array}$ & $\begin{array}{c}\text { Frequency } \\
\text { Repetition }\end{array}$ & $\begin{array}{c}\text { Receiver } \\
\text { Sensitivity } \\
\mathbf{( d B m})\end{array}$ & $\begin{array}{c}\text { Effective } \\
\text { Data-Rate } \\
\text { (kbps) }\end{array}$ & $\begin{array}{c}\text { Channel } \\
\text { Bandwidth } \\
\text { (kHz) }\end{array}$ & Abbreviation \\
\hline $\begin{array}{c}\text { OQPSK- } \\
\text { DSSS }\end{array}$ & N/A & OQPSK & N/A & N/A & -103 & 250 & 5000 & OQPSK-DSSS \\
\hline $\begin{array}{c}\text { OFDM } \\
\text { Option 1 }\end{array}$ & MCS1 & BPSK & $1 / 2$ & $2 x$ & -109 & 200 & 1200 & OFDM1-MCS1 \\
\hline $\begin{array}{c}\text { OFDM } \\
\text { Option 2 }\end{array}$ & MCS2 & QPSK & $1 / 2$ & $2 x$ & -108 & 200 & 800 & OFDM2-MCS2 \\
\hline $\begin{array}{c}\text { OFDM } \\
\text { Option 3 }\end{array}$ & MCS3 & QPSK & $1 / 2$ & $0 x$ & -107 & 200 & 400 & OFDM3-MCS3 \\
\hline $\begin{array}{c}\text { OFDM } \\
\text { Option 4 }\end{array}$ & MCS5 & 16-QAM & $1 / 2$ & $0 x$ & -105 & 200 & 200 & OFDM4-MCS5 \\
\hline
\end{tabular}

For the measurement campaign, each selected modulation is used as an interferer signal, and tested against all other selected modulations acting as transmitter signals (OFDM1-MCS1, OFDM2-MCS2, OFDM3-MCS3, OFDM4-MCS5, OQPSK-DSSS). The procedure is also repeated for two different payload lengths ( 20 bytes and 120 bytes) in order to understand the effects of packet length on the robustness of each modulation. This is relevant as the SUN-OFDM modulation adds symbol redundancy in both time and frequency, whereas OQPSK-DSSS only adds redundancy in terms of the spreading factor gain. In addition, each base experiment is repeated twice for each transmitter and interferer device (i.e., exchanging the devices' roles). We use this approach to verify the obtained results and cancel out possible differences in the transmit power between the devices (due to the radio transceiver construction variability and/or the impedance matching of the RF circuit) that may affect the obtained results.

Overall, this gives a total of 10 base experiments, one for each interferer signal and payload length, as presented in Section 5. Each base experiment uses a particular modulation as interferer, and evaluates the robustness of all the modulations being tested in the measurement campaign, including itself. For instance, OFDM1-MCS1 is selected as interferer and tested against OFDM1-MCS1, OFDM2-MCS2, OFDM3-MCS3, OFDM4-MCS5 and OQPSK-DSSS.

To ensure that noise and external interference do not affect the results, we set the transmit power to a value that is well above the sensitivity limit of the radio transceiver for each modulation 
being tested, and conduct the experiments using RF coaxial cables while each board is enclosed in an individual Faraday cage. Second, when both transmitter and interferer devices use the same modulation, it is possible that the receiver synchronizes to the header transmitted by the interferer. To avoid this, the interferer device uses continuous transmit mode and starts transmitting $10 \mathrm{~ms}$ before the transmitter device starts sending data packets.

\subsection{Base Experiment}

The independent variable of each base experiment is the SIR (Signal-to-Interference Ratio), defined as the ratio between the power of the transmitter signal and the power of the interferer signal; thermal noise is not taken into account since both signals are well-above its value $\left(P_{N}=-107 \mathrm{dBm}\right)$ considering the experiment conditions, i.e., ambient temperature $\left(\mathrm{T}=25^{\circ} \mathrm{C}\right)$ and signal bandwidth $(\mathrm{B} \leq 5 \mathrm{MHz})$. In contrast, the dependent variable of each experiment is the PDR (Packet Delivery Ratio), defined as the percentage of packets that are successfully received at the receiver under the presence of the interference signal generated by the interferer device.

For all the experiments, the value of the SIR ranges from $-12 \mathrm{~dB}$ to $+6 \mathrm{~dB}$, meaning that the transmitter power is between $1 / 16 \times$ and $4 \times$ the interferer power. We use such a wide SIR range to ensure that the PDR transitions from $100 \%$ to $0 \%$ regardless of the interferer and transmitter modulation under test. In addition, to validate the results obtained from each base experiment, the first test is conducted without interference signal (the interferer device does not transmit during that test). In that case, the PDR is expected to be $100 \%$. This can be observed with the Inf mark on the $x$-axis of the figures presented in Section 5.

To determine the PDR for each SIR for a given transmit modulation, the transmitter device sends 1000 frames to the receiver device. Frames are transmitted with a $5 \mathrm{~ms}$ inter-packet delay. The payload is composed of the $\{0 x 00,0 x 01, \ldots, 0 x 13\}$ sequence for the 20-bytes payload and the $\{0 x 00,0 x 01, \ldots, 0 x 77\}$ sequence for the 120-bytes payload. Each frame is completed with the FCS (Frame Check Sequence) field of the PSDU depending on the selected modulation (2 bytes for OQPSK-DSSS, 4 bytes for SUN-OFDM).

During the transmission, the interferer device injects an inteferent signal using the selected modulation for that particular base experiment. In contrast to data packets, the payload of the interference packets is composed of a 123-bytes $\{0 x 55, \ldots, 0 \times 55\}$ sequence. That payload is transmitted continuously using the continuous transmission feature of the radio transceiver. That is, the transceiver operates in packet mode, but the packet header (SHR + PHR) is only transmitted once and the payload (PSDU + FCS) is transmitted indefinitely until the experiment is finished.

There are two conditions that need to be met for a packet to be considered successfully received. First, the FCS calculated by the receiver radio transceiver based on the received bytes has to match the FCS value attached by the transmitter device at the end of the payload. Second, the received bytes have to match the transmitted bytes depending on the packet length for that base experiment. This ensures that the PDR results are correct even in the unexpected case where the calculated FCS sequence matches the received FCS sequence despite one or multiple bit errors caused by the interferer signal.

\subsection{Setup}

For the transmitter, receiver and interferer devices we use the OpenMote-B $[16,17]$ shown in Figure 4. The OpenMote-B is equipped with a Texas Instruments CC2538 SoC (System on Chip) and a Microchip AT86RF215 radio transceiver. The CC2538 [18] includes an ARM Cortex-M3 micro-controller ( $32 \mathrm{MHz}, 32 \mathrm{kB}$ RAM, $512 \mathrm{kB}$ Flash) and a radio transceiver compatible with the IEEE 802.15.4-2006 standard. The AT86RF215 [19] is a dual-band (sub-GHz and $2.4 \mathrm{GHz}$ ) radio transceiver compatible with the IEEE 802.15.4-2015 standard, which supports all Multi-Rate PHY options defined in the IEEE 802.15.4g-2012 amendment for Smart Utility Networks (SUN). 


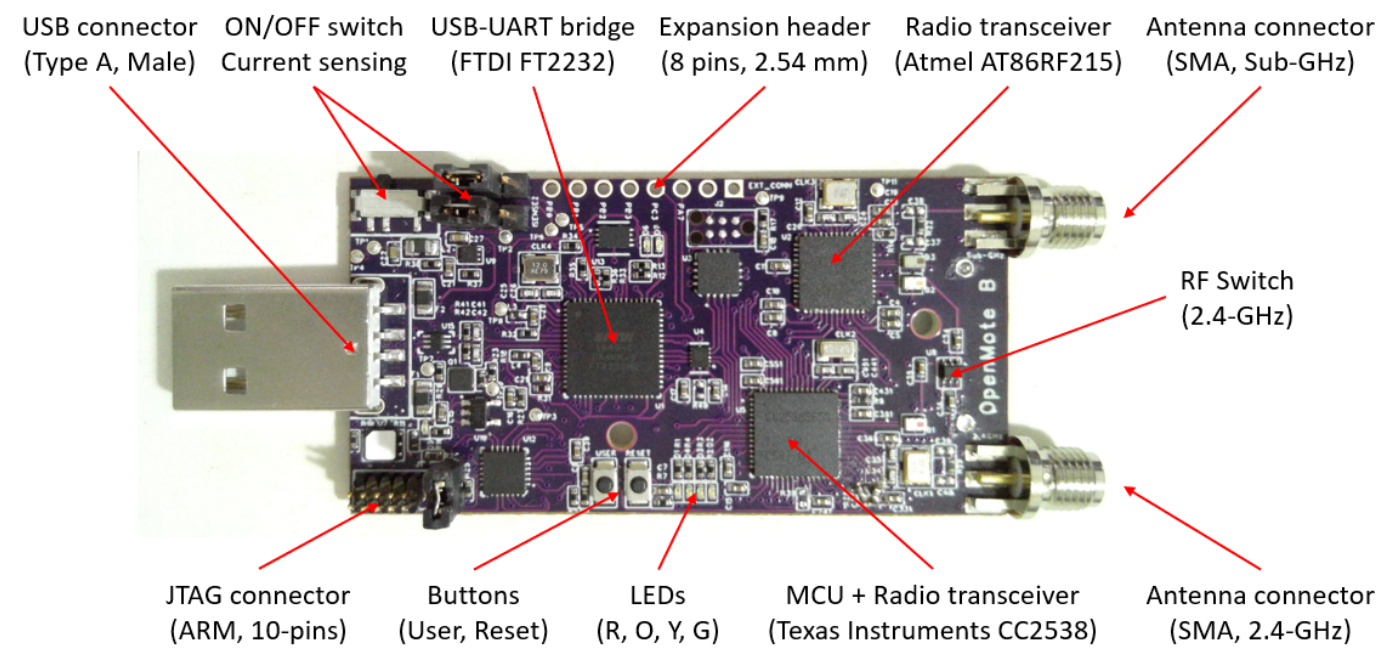

Figure 4. OpenMote-B board. The CC2538 (MCU) and the AT86RF215 (radio transceiver) are interconnected using the SPI bus, whereas the CC2538 (MCU) is connected to the computer using the USB-UART bridge (FTDI FT2232).

As depicted in Figure 3, the boards are connected using an Agilent 87302C RF power combiner that operates in the $0.5-26.5 \mathrm{GHz}$ band and provides an input attenuation of $3 \mathrm{~dB}$ for the input channels towards the output channel, and a return loss of $24 \mathrm{~dB}$. The output of the RF power combiner is further attenuated using a Minicircuit $30 \mathrm{~dB}$ RF attenuator to ensure that the received signal at the receiver device is within the receive power limits of the AT86RF215 radio transceiver $(-5 \mathrm{dBm}$ according to the data-sheet).

To coordinate the actions between the different devices, a computer running Ubuntu 18.04 LTS is used. The computer is connected to the OpenMote-B boards over USB, and executes a Python script that orchestrates an experiment. For each board, the script uploads the radio configuration to be used (i.e., modulation type, transmit power and packet length, among others) and starts transmission/reception. The OpenMote-B boards execute a custom firmware that receives commands over UART (Universal Asynchronous Receiver Transmitter), execute the action commanded (i.e., transmit a packet, start receiving, etc.) and return the result (i.e., packet successfully received or not).

\subsection{Radio Calibration}

The PDR is evaluated with respect to SIR between the transmit power of the data and the interferer signals. According to the AT86RF215 radio transceiver datasheet (specifically Figure 11-2), there is a $6 \mathrm{~dB}$ offset between the value of the transmit power configuration register (RFn_PAX. TXPWR) and the actual transmit power of the radio transceiver depending on the selected modulation (OQPSK-DSSS and SUN-OFDM).

Such difference in transmit power between different modulations can have an impact on the obtained results. For instance, an uncontrolled higher transmit power of the data signal results in a better PDR result for that particular modulation. Similarly, an uncontrolled lower transmit power of the interferer signal also results in a better PDR for that particular modulation. To validate the offset and obtain the configuration register values that ensure the same transmit power for both modulations, we calibrate using a Rigol DSA-815 spectrum analyzer as the Test Equipment (TE), and an OpenMote-B as the Device Under Test (DUT).

The DUT is connected to TE using an RF cable and the radio calibration procedure is performed as follows. For each modulation, the DUT is configured to transmit with a known power configuration register and the TE is configured to measure the power in the band taking into consideration the parameters of the modulation under test (i.e., center frequency, occupied bandwidth and span). The procedure is repeated for all the values of the power configuration register (from 0 to 30 with 
a 3-unit step) to obtain the relation between those values and the actual transmit powers that are generated by the radio transceiver, as shown in Table 3.

Table 3. Transmit power values for the OQPSK-DSSS and the SUN-OFDM modulations. Notice that the results have been rounded to the closest $\mathrm{dB}$ given the resolution of the DUT (Device Under Test) and the uncertainty of the TE (Test Equipment).

\begin{tabular}{cccccc}
\hline \multicolumn{7}{c}{ Transmit Power (dBm) } \\
\hline RFn_PAX.TXPWR & OQPSK-DSSS & OFDM1-MCS1 & OFDM2-MCS2 & OFDM3-MCS3 & OFDM4-MCS54 \\
\hline $\mathbf{3 0}$ & 15 & 9 & 9 & 9 & 9 \\
\hline $\mathbf{2 7}$ & 14 & 8 & 8 & 8 & 8 \\
\hline $\mathbf{2 4}$ & 12 & 5 & 5 & 5 & 5 \\
\hline $\mathbf{2 1}$ & 9 & 2 & 2 & 2 & -1 \\
\hline $\mathbf{1 8}$ & 6 & -1 & -1 & -3 & -1 \\
\hline $\mathbf{1 5}$ & 3 & -3 & -3 & -7 & -3 \\
\hline $\mathbf{1 2}$ & 0 & -7 & -7 & -11 & -11 \\
\hline $\mathbf{9}$ & -3 & -11 & -11 & -14 & -14 \\
\hline $\mathbf{6}$ & -6 & -14 & -14 & -16 & -16 \\
\hline $\mathbf{3}$ & -9 & -16 & -16 & -18 & -18 \\
\hline $\mathbf{0}$ & -12 & -18 & -18 & & -16 \\
\hline
\end{tabular}

Overall, results are in accordance with the data provided in the datasheet and allow for empirically determining the transmit power configuration register values for the OQPSK-DSSS and the SUN-OFDM modulations. In particular, we have decided to use a transmit power between $-9 \mathrm{dBm}$ and $+9 \mathrm{dBm}$, as these are the values that are common to both modulations. We then define the default transmit power of $+3 \mathrm{dBm}$, and let the interferer power range from $-9 \mathrm{dBm}$ to $+9 \mathrm{dBm}$, corresponding to the SIR between $-12 \mathrm{~dB}$ and $+6 \mathrm{~dB}$ described earlier.

\section{Results}

Results obtained using the research methodology and the experiment setup described in the previous section are presented for each interferer modulation (OFDM1-MCS1, OFDM2-MCS2, OFDM3-MCS3, OFDM4-MCS5 and OQPSK-DSSS) and packet length (20 bytes and 120 bytes) in Figures 5-9, respectively. Table 4 summarizes the required SIR (dB) to ensure PDR $>80 \%$ for each modulation and packet length.

Table 4. Results summary. Each value in the table is the required SIR (dB) to ensure a PDR $>80 \%$ for each modulation (OFDM1-MCS1, OFDM2-MCS2, OFDM3-MCS3, OFDM4-MCS5, OQPSK-DSSS) and packet length $(20,120$ bytes).

\begin{tabular}{lcccccccccc}
\hline & \multicolumn{1}{c}{ Interference Type and Packet Length (bytes) } \\
\cline { 2 - 15 } & \multicolumn{1}{c}{ OFDM1-MCS1 } & \multicolumn{1}{c}{ OFDM2-MCS2 } & OFDM3-MCS3 & OFDM4-MCS5 & \multicolumn{2}{c}{ OQPSK-DSSS } \\
\cline { 2 - 13 } & $\mathbf{2 0}$ & $\mathbf{1 2 0}$ & $\mathbf{2 0}$ & $\mathbf{1 2 0}$ & $\mathbf{2 0}$ & $\mathbf{1 2 0}$ & $\mathbf{2 0}$ & $\mathbf{1 2 0}$ & $\mathbf{2 0}$ & $\mathbf{1 2 0}$ \\
\hline OFDM1-MCS1 & 1 & 2 & 2 & 3 & 3 & 4 & 2 & 3 & -1 & -1 \\
\hline OFDM1-MCS2 & 2 & 4 & 5 & 6 & 5 & 6 & 5 & 7 & -5 & -3 \\
\hline OFDM3-MCS3 & 2 & 4 & 5 & 6 & 8 & 9 & 8 & 9 & -5 & -3 \\
\hline OFDM4-MCS5 & 5 & 7 & 8 & 10 & 10 & 12 & $>12$ & $>12$ & -2 & 0 \\
\hline OQPSK-DSSS & 8 & 12 & 8 & 12 & 8 & 12 & 9 & 12 & 6 & 8 \\
\hline
\end{tabular}




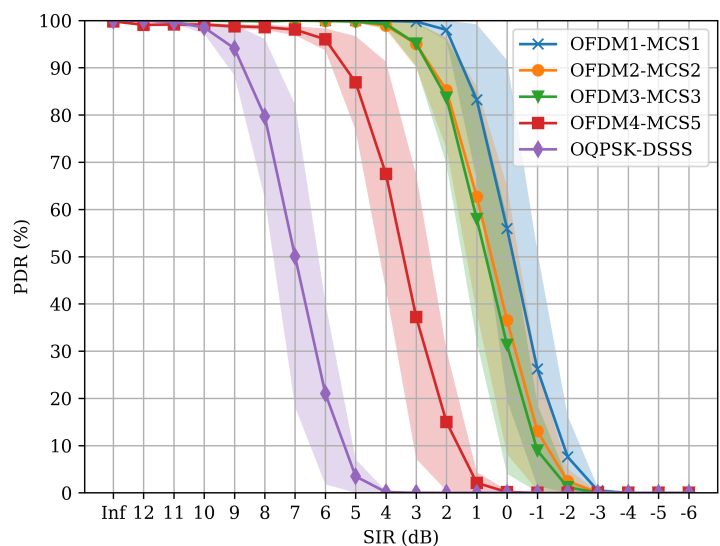

(a) PSDU $=20$ bytes

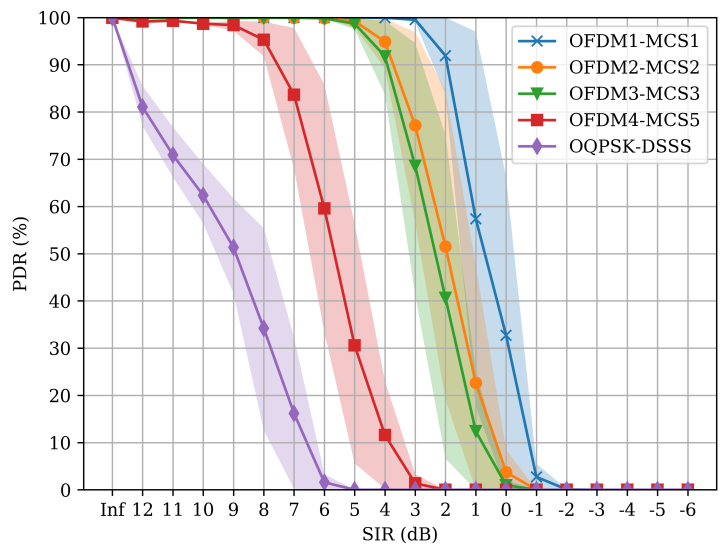

(b) PSDU $=120$ bytes

Figure 5. OFDM1-MCS1 interference results.

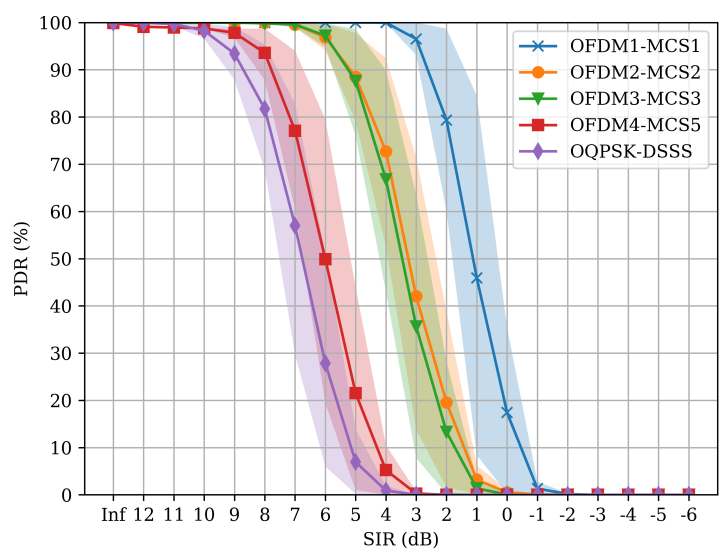

(a) PSDU $=20$ bytes

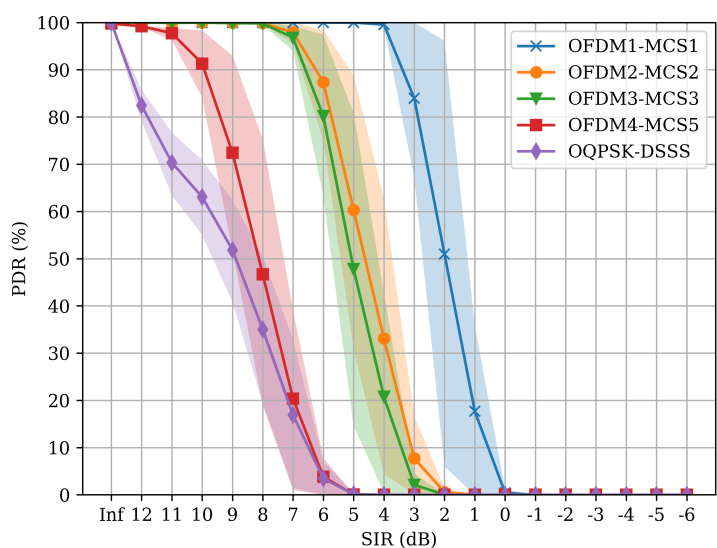

(b) PSDU $=120$ bytes

Figure 6. OFDM2-MCS2 interference results.

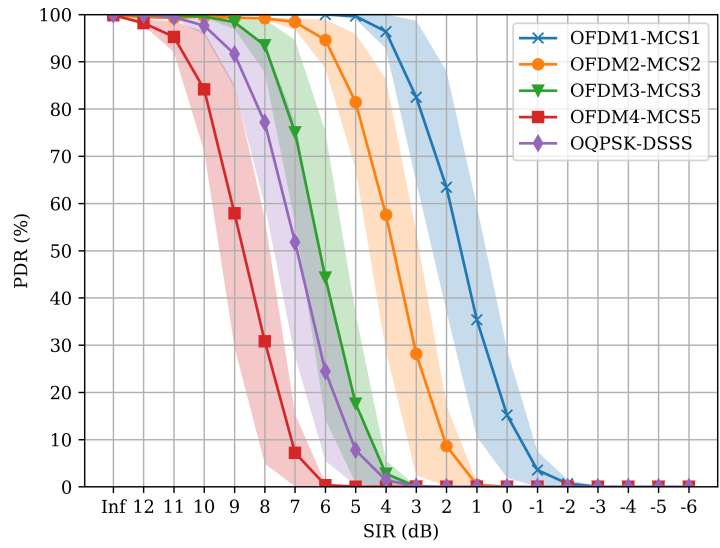

(a) PSDU $=20$ bytes

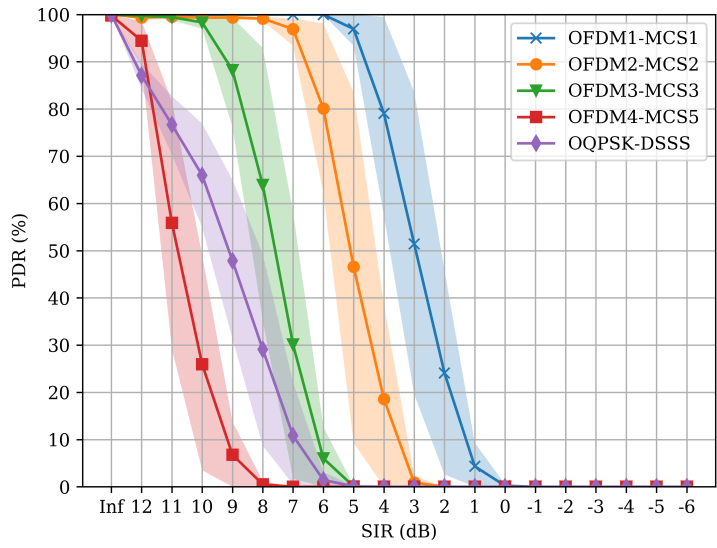

(b) PSDU $=120$ bytes

Figure 7. OFDM3-MCS3 interference results. 


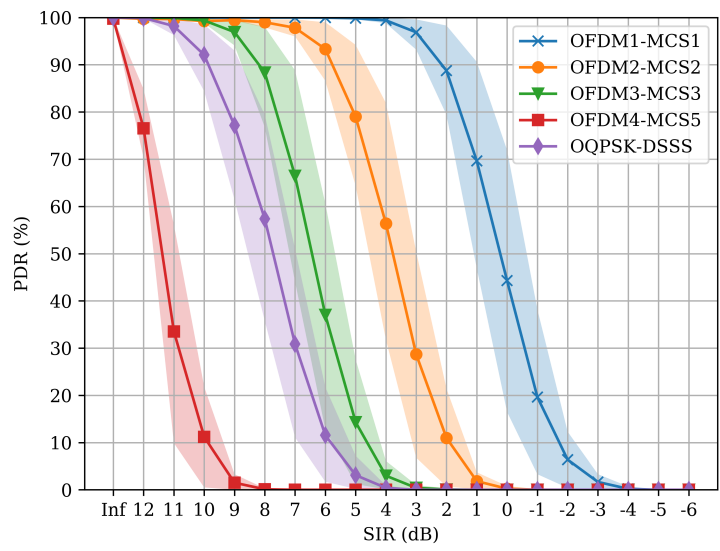

(a) PSDU $=20$ bytes

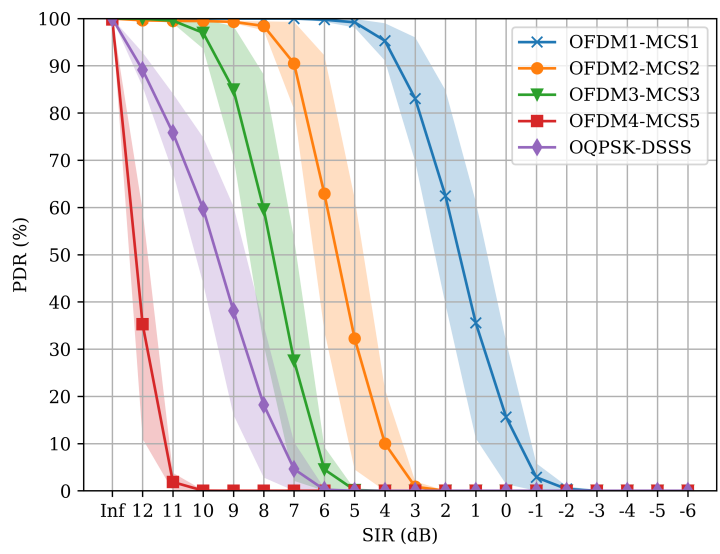

(b) PSDU $=120$ bytes

Figure 8. OFDM4-MCS5 interference results.

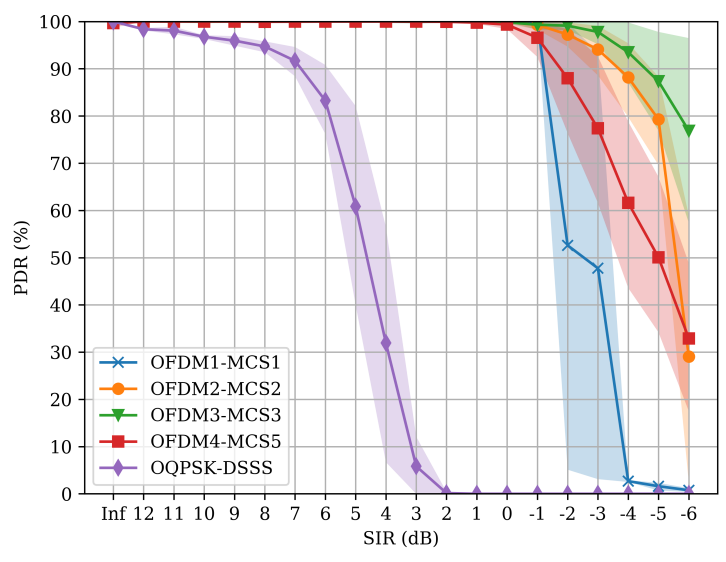

(a) PSDU $=20$ bytes

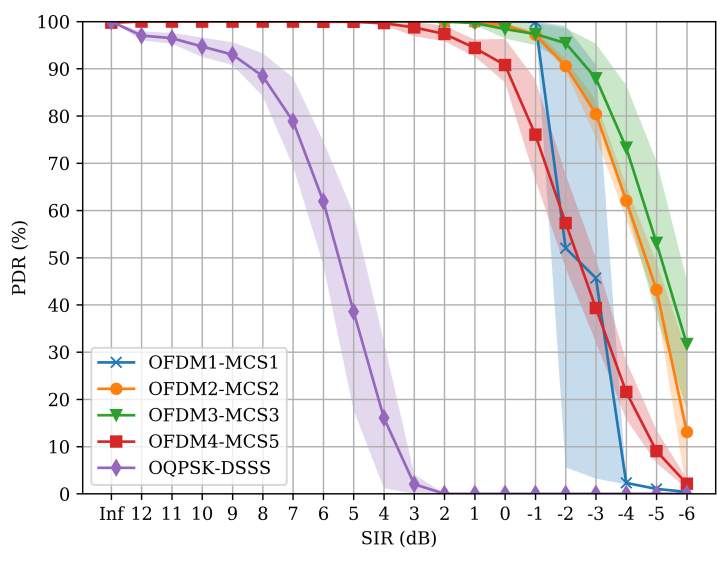

(b) PSDU $=120$ bytes

Figure 9. OQPSK-DSSS interference results.

For example, if we consider an interfering signal modulated using OFDM1-MCS1, we can observe that, for a 20 bytes PSDU, the OFDM1-MCS1, OFDM2-MCS2 and OFDM-MCS3 physical layers perform similarly, requiring SIR $>1 \mathrm{~dB}$ for PDR $>80 \%$. OFDM4-MCS5 requires SIR $>5 \mathrm{~dB}$ to provide a PDR $>80 \%$. In contrast, for a 120 B PSDU, OFDM1-MCS1 requires an SIR $>2 \mathrm{~dB}$, OFDM2-MCS2 and OFDM3-MCS3 require SIR $>4 \mathrm{~dB}$, and OFDM4-MCS5 requires an SIR $>7 \mathrm{~dB}$. Finally, for a 20 byte and 120 byte PSDU, OQPSK-DSSS requires an SIR $>8 \mathrm{~dB}$ and an SIR $>12 \mathrm{~dB}$ for PDR $>80 \%$, respectively.

These results show that OQPSK-DSSS requires $6 \mathrm{~dB}$ higher SIR to achieve PDR $>80 \%$ when compared to OFDM1-MCS1 under the same interference. Considering that OQPSK-DSSS uses a $5 \mathrm{MHz}$ channel bandwidth while OFDM1-MCS1 uses $1.2 \mathrm{MHz}$ channel bandwidth, we can derive that OFDM provides increased spectral efficiency and robustness. Similar results can be derived for other combinations, as presented in Table 4.

Interestingly, considering an interfering signal modulated using OQPSK-DSSS, if we consider a system using OFDM1-MCS1 and we compare it to the same system using OQPSK-DSSS, we can observe that OFDM1-MCS1 can still operate with PDR $>80 \%$ even if the interference level is $1 \mathrm{~dB}$ above the signal level. This is particularly interesting because OQPSK-DSSS is widely used. 


\section{Discussion and Recommendations}

This section discusses the results outlined in the previous section and presents recommendations to researchers and practitioners, serving as a tool to understand the behavior of each particular modulation under interference conditions, and motivating the use of the SUN-OFDM for deploying low-power wireless systems in industrial scenarios.

\subsection{Discussion}

In the following, we discuss the results presented in Table 4.

First, considering OFDM1-MCS1, we observe that a reduction in the bandwidth of the interference signal (i.e., going from OFDM2-MCS2 to OFDM4-MCS5 interference) requires a $2 \mathrm{~dB}$ SIR increase to provide a PDR $>80 \%$. This can be attributed to the fact that the reduction in the bandwidth causes an increase in the PSD (Power Spectral Density) of the interference (i.e., a $3 \mathrm{~dB}$ increase each time the bandwidth is reduced by half) meaning that the probability of a symbol being corrupted increases.

Second, the SIR required for OFDM2-MCS2 and OFDM3-MCS3 is equivalent to OFDM1-MCS1 and OFDM2-MCS2 regardless of packet size (2-4 dB and 5-6 dB, resp.). However, the effect of OFDM3-MCS3 interference causes a $3 \mathrm{~dB}$ SIR increase to the OFDM3-MCS3 signal (i.e., 7-8 dB) to maintain PDR $>80 \%$, whereas the OFDM2-MCS2 signal remains unaffected (5-6 dB). Such results can be attributed to the fact that, despite both OFDM2-MCS2 and OFDM3-MCS3 using the same modulation (OQPSK with coding rate $R=1 / 2$ ), the former takes advantage of the $2 x$ frequency repetition providing an additional protection of $3 \mathrm{~dB}$. The result is independent of packet length, which confirms the importance of using such protection mechanisms at the physical layer.

Third, the SIR required for OFDM4-MCS5 to maintain a PDR $>80 \%$ is higher compared to the other SUN-OFDM modulations and increases as the bandwidth of the interferer is reduced (going from OFDM1-MCS1 to OFDM4-MCS5 interference). This is an expected result, as the PSD of the interference increases by $3 \mathrm{~dB}$ every time the bandwidth is reduced by two, and taking into account that OFDM4-MCS5 uses an aggressive modulation (16-QAM) in combination with a coding rate $R=1 / 2$, but does not employ time or frequency symbol repetition, leading to a higher BER (Bit Error Rate) in the presence of interference.

Fourth, the SIR required for the OQPSK-DSSS modulation remains constant ( $8-12 \mathrm{~dB}$ depending on packet length) regardless of the type of the SUN-OFDM interference and, hence, the occupied bandwidth. In contrast, OQPSK-DSSS modulation requires a smaller SIR (6-8 dB depending on packet length) when the interference is OQPSK-DSSS. This can be attributed to a combination of facts including that SUN-OFDM interference is composed of multiple pilots spread over the channel and has a higher PSD, as well as the fact that OQPSK-DSSS does not provide any additional mechanism to enhance the robustness of the transmission against interference except for the processing gain of DSSS $\left(10 \cdot \log _{10}(2 \mathrm{M} / 250 \mathrm{k})=9 \mathrm{~dB}\right)$.

Fifth, SUN-OFDM modulations are robust against OQPSK-DSSS interference, requiring negative SIR values (SIR $<0 \mathrm{~dB}$ ) to maintain a PDR $>80 \%$. This is an expected result that can be attributed to the fact that SUN-OFDM modulation is a combination of narrowband modulation, whereas the OQPSK-DSSS is inherently wideband, i.e., it has a very low PSD. In fact, the results show that OFDM2-MCS2 and OFDM3-MCS3 require the same SIR, which is lower than OFDM1-MCS1. This can be explained given that OFDM2-MCS2 employs $2 \times$ frequency repetition, whereas OFDM3-MCS3 occupies half the bandwidth ( $400 \mathrm{kHz}$ vs. $800 \mathrm{kHz}$, resulting in a $3 \mathrm{~dB}$ PSD increase).

As a numeric example of the above, for OFDM2-MCS2, the PSD for a transmit power of $0 \mathrm{dBm}$ and a bandwidth of $800 \mathrm{kHz}$ is $-29.03 \mathrm{dBm} / \mathrm{Hz}$. In contrast, for OFDM3-MCS3, the PSD for a transmit power of $0 \mathrm{dBm}$ and a bandwidth of $400 \mathrm{kHz}$ is $-26.02 \mathrm{dBm} / \mathrm{Hz}$. Finally, OQPSK-DSSS interference with a transmit power of $0 \mathrm{dBm}$ and a bandwidth of $2 \mathrm{MHz}$ has a PSD of $-33.01 \mathrm{dBm} / \mathrm{Hz}$. Notice that, for an SIR $=0 \mathrm{~dB}$, OFDM2-MCS2 has SIR $=4 \mathrm{~dB}(-29.03--33.01=3.98 \mathrm{~dB})$, whereas OFDM3-MCS3 has $\operatorname{SIR}=7 \mathrm{~dB}(-26.02--33.01=6.99 \mathrm{~dB})$. In conclusion, when dealing with wideband interference 
(here, OQPSK-DSSS), using $2 \times$ repetition provides a theoretical $3 \mathrm{~dB}$ advantage, but that advantage is overcome by the bandwidth reduction that provides a higher PSD.

Another interesting result comparing the performance of the SUN-OFDM and OQPSK-DSSS physical layers against interference is that the degradation in terms of PDR caused by the increment of the packet length (going from 20 bytes to 120 bytes) is not equal for both modulation types. For SUN-OFDM, the SIR increase to maintain a PDR $>80 \%$ is bounded to $2 \mathrm{~dB}$, whereas, for OQPSK-DSSS, the SIR increase is larger than $3 \mathrm{~dB}$. This is thanks to time and frequency diversity, which allows for recovering random errors caused by multi-path propagation and external interference.

The SIR spread between operating in ideal conditions (PDR $>80 \%$ ) and degraded conditions (PDR $<20 \%$ ) for all SUN-OFDM modulations requires SIR $=3 \mathrm{~dB}$ regardless of the packet length. In contrast, the SIR spread between operating in ideal conditions and degraded conditions for OQPSK-DSSS depends on packet size and interference type. When dealing with SUN-OFDM interference, transitions require SIR $=2 \mathrm{~dB}$ for 20-byte packets, and $\mathrm{SIR}=5 \mathrm{~dB}$ for 120-byte packets. In contrast, when dealing with OQPSK-DSSS interference, the transition requires SIR $=2 \mathrm{~dB}$ regardless of packet length.

Comparing OFDM1-MCS1 to OQPSK-DSSS, we observe that, for a PDR $>80 \%$, it provides an advantage between 5-10 dB, depending on interference type and packet length. In particular, it provides an advantage of $5 \mathrm{~dB}$ for OFDM3-MCS3 interference with packet length of 20 bytes, and an advantage of $10 \mathrm{~dB}$ for OFDM1-MCS1 interference with packet length of 120 bytes. This is in contrast with the fact that OFDM1-MCS1 uses a channel separation of $1.2 \mathrm{MHz}$, whereas OQPSK-DSSS uses a channel separation of $5 \mathrm{MHz}$. That is, the spectral efficiency of OFDM1-MCS1 is $0.166 \mathrm{bits} / \mathrm{Hz}$ (or $200 \mathrm{kbps} / 1200 \mathrm{kHz}$ ), whereas the spectral efficiency of OQPSK-DSSS is $0.05 \mathrm{bits} / \mathrm{Hz}$ (or $250 \mathrm{kbps} / 5000 \mathrm{kHz}$ ). Hence, OFDM1-MCS1 provides an increased robustness level while offering a higher spectral efficiency that enables having denser deployments (a 333\% capacity increase).

From these results, we can observe that the SIR required to provide PDR $>80 \%$ for OQPSK-DSSS largely depends on the packet length ( $4 \mathrm{~dB}$ difference). In contrast, the SIR required to provide a PDR $>80 \%$ for the robust SUN-OFDM modulations remains constant $(<1 \mathrm{~dB})$ regardless of the packet length and the interference type. This is owing to the fact that OQPSK-DSSS does not use any physical layer mechanism to enhance robustness except for the processing gain provided by DSSS. Hence, the error probability increases as the packet length is increased. For SUN-OFDM-based modulations, time and frequency symbol repetition reduces the probability of having an erroneous bit.

\subsection{Recommendations}

Based on the results and the discussion presented above, we provide the following insights and recommendations to researchers and practitioners deploying low-power wireless communication systems using the SUN-OFDM physical layer in real-world environments:

1. Thanks to the small effect of packet length in the PDR with respect to the SIR, SUN-OFDM allows for using larger packets (i.e., packet bundling) to increase the transmission efficiency (i.e., more effective data with the same packet headers) without sacrificing robustness. In fact, SUN-OFDM allows payloads of up to 2047 bytes, effectively allowing to transmit full IPv6 packets without fragmentation or allowing to group up to sixteen 127-bytes frames in a single 2047-bytes frame.

2. Despite the fact that SUN-OFDM transceivers consume a higher amount of energy compared to state-of-the-art IEEE 802.15.4 transceivers due to the additional circuitry required to operate (scrambler, convolutional encoder, puncturer, interleaver, Viterbi decoder), the higher level of robustness against interference provided by SUN-OFDM allows for using higher data rates (up to $800 \mathrm{kbps}$ ) to reduce the average energy consumption of the transmitter and the receiver devices.

3. As the preamble of a SUN-OFDM packet is transmitted using the lowest MCS option of the current configuration, and includes information regarding the MCS option used to transmit the packet payload, this allows the transmitter to switch between different MCS options of the same SUN-OFDM configuration without any changes in the receiver configuration. It is hence possible for the transmitter to use an aggressive modulation for an initial packet transmission, and use 
a more robust modulation when re-transmitting. Similarly, acknowledgement frames can be transmitted using the most robust modulation to increase the probability they are received.

4. The use of SUN-OFDM for deployments with a high device density and/or high interference levels is advisable, as it provides higher spectral efficiency, while maintaining a similar level of robustness against interference with respect to OQPSK-DSSS. If interference is of concern, choosing OFDM1-MCS1 over OQPSK-DSSS translates into a 4x capacity increase while providing an average advantage of $9 \mathrm{~dB}$ against the same interference. In contrast, if interference is not a concern, choosing OFDM4-MCS5 offers a 26x capacity increase, while maintaining a similar level of protection against the same type of interference.

\section{Conclusions}

This article evaluates the interference robustness of the OQPSK-DSSS and the SUN-OFDM physical layers defined in the IEEE 802.15.4-2015 standard. Interfering conditions are generated in a controlled setup to evaluate the SIR required by each modulation to achieve PDR $>80 \%$. Compared to OQPSK-DSSS, results show that SUN-OFDM provides at least $6 \mathrm{~dB}$ additional protection regardless of interference type and packet length. In addition, SUN-OFDM only occupies $1.2 \mathrm{MHz}$ bandwidth, whereas OQPSK-DSSS occupies $5 \mathrm{MHz}$. This results in a higher spectral efficiency that allows one to have denser deployments or to trade bandwidth efficiency and interference robustness depending on the application requirements. Overall, the presented results become a useful tool to understand the behavior of each particular modulation under interference conditions, and motivates the use of the SUN-OFDM physical layer to deploy low-power wireless systems in industrial scenarios.

Author Contributions: P.T.-P. developed the firmware/software, conducted the experiments, obtained the graphics and contributed to writing the manuscript. F.V.-G. and J.M. equally contributed to conceptualizing the experiments, validating the results and writing the manuscript. T.W., J.A.-Z., and X.V. equally contributed to the supervision of the projects and the review of the manuscript.

Funding: This research is partially supported by Generalitat de Catalunya (SGR-60-2017 and SGR-891-2017) and the Spanish Ministry of Science, Innovation and Universities (SPOT5G <TEC2017-87456-P> and SPOTS $<$ RTI2018-095438-A-I00>).

Conflicts of Interest: The authors declare no conflict of interest.

\begin{tabular}{|c|c|}
\hline \multicolumn{2}{|c|}{ Abbreviations } \\
\hline The followin & abbreviations are used in this manuscript: \\
\hline BPSK & Binary Phase Shift Keying \\
\hline CSMA/CA & Carrier Sense Multiple Access/Collision Avoidance \\
\hline DSSS & Direct Sequence Spread Spectrum \\
\hline DUT & Device Under Test \\
\hline ED & Energy Detection \\
\hline FCS & Frame Check Sequence \\
\hline FDMA & Frequency Division Multiple Access \\
\hline FSK & Frequency Shift Keying \\
\hline IEEE & Institute of Electrical and Electronic Engineers \\
\hline ISM & Industrial, Scientific and Medical \\
\hline LoRa & Long Range \\
\hline LP-WAN & Low-Power Wide-Area Network \\
\hline MAC & Medium Access Control \\
\hline MCS & Modulation and Coding Scheme \\
\hline MCU & Micro-Controller Unit \\
\hline OFDM & Orthogonal Frequency Division Multiplexing \\
\hline
\end{tabular}




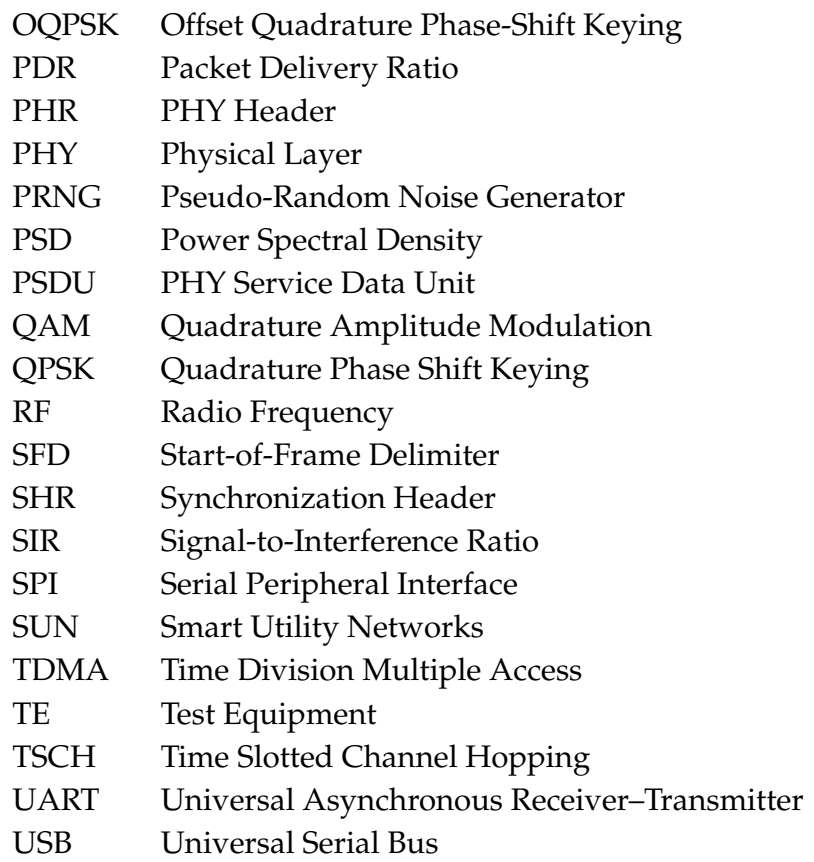

\section{References}

1. IEEE Standard for Low-Rate Wireless Networks; IEEE Std 802.15.4-2015 (Revision of IEEE Std 802.15.4-2011); IEEE: Piscataway, NJ, USA, 2016; pp. 1-709. [CrossRef]

2. Gutierrez, J.A.; Naeve, M.; Callaway, E.; Bourgeois, M.; Mitter, V.; Heile, B. IEEE 802.15.4: A developing standard for low-power low-cost wireless personal area networks. IEEE Netw. 2001, 15, 12-19. [CrossRef]

3. Gislason, D. Zigbee Wireless Networking; Newnes: Cambridge, MA, USA, 2008.

4. ANSI/ISA-100.11a-2011 Wireless Systems for Industrial Automation: Process Control and Related Applications; International Society of Automation Standard: Durham, NC, USA, 2011.

5. Chen, D.; Nixon, M.; Mok, A. WirelessHART: Real-Time Mesh Network for Industrial Automation, 1st ed.; Springer Publishing Company: New York, NY, USA, 2010.

6. Vilajosana, X.; Watteyne, T.; Vučinić, M.; Chang, T.; Pister, K.S.J. 6TiSCH: Industrial Performance for IPv6 Internet-of-Things Networks. Proc. IEEE 2019, 1153-1165. [CrossRef]

7. IEEE Standard for Local and Metropolitan Area Networks-Part 15.4: Low-Rate Wireless Personal Area Networks (LR-WPANs) Amendment 1: MAC Sublayer; IEEE Std 802.15.4e-2012 (Amendment to IEEE Std 802.15.4-2011); IEEE: Piscataway, NJ, USA, 2012; pp. 1-225, doi:10.1109/IEEESTD.2012.6185525.

8. IEEE Standard for Local and Metropolitan Area Networks-Part 15.4: Low-Rate Wireless Personal Area Networks (LR-WPANs) Amendment 3: Physical Layer (PHY) Specifications for Low-Data-Rate, Wireless, Smart Metering Utility Networks; IEEE Std 802.15.4g-2012 (Amendment to IEEE Std 802.15.4-2011); IEEE: Piscataway, NJ, USA, 2012; pp. 1-252.

9. McCune, E. This Emperor Has No Clothes? IEEE Microwaves Mag. 2013, 14, 48-62. [CrossRef]

10. Sommer, P.; Maret, Y.; Dzung, D. Low-Power Wide-Area Networks for Industrial Sensing Applications. In Proceedings of the 2018 IEEE International Conference on Industrial Internet (ICII), Seattle, WA, USA, 21-23 October 2018; pp. 23-32. [CrossRef]

11. Sum, C.; Kojima, F.; Harada, H. Coexistence of homogeneous and heterogeneous systems for IEEE 802.15.4g smart utility networks. In Proceedings of the 2011 IEEE International Symposium on Dynamic Spectrum Access Networks (DySPAN), Aachen, Germany, 3-6 May 2011; pp. 510-520. [CrossRef]

12. Liu, Y.; Guo, J.; Orlik, P.; Nagai, Y.; Watanabe, K.; Sumi, T. Coexistence of 802.11ah and 802.15.4g networks. In Proceedings of the 2018 IEEE Wireless Communications and Networking Conference (WCNC), Barcelona, Spain, 15-18 April 2018; pp. 1-6. [CrossRef]

13. Munoz, J.; Chang, T.; Vilajosana, X.; Watteyne, T. Evaluation of IEEE802.15.4g for Environmental Observations. Sensors 2018, 18, 3468. [CrossRef] [PubMed] 
14. Munoz, J.; Riou, E.; Vilajosana, X.; Muhlethaler, P.; Watteyne, T. Overview of IEEE802.15.4g OFDM and its Applicability to Smart Building Applications. In Proceedings of the Wireless Days Conference (WD), Dubai, UAE, 3-5 April 2018; IEEE: Piscataway, NJ, USA, 2018; doi:10.1109/WD.2018.8361707. [CrossRef]

15. Munoz, J.; Muhlethaler, P.; Vilajosana, X.; Watteyne, T. Why Channel Hopping Makes Sense, even with IEEE802.15.4 OFDM at 2.4 GHz. In Proceedings of the Global IoT Summit (GIoTS), Bilbao, Spain, 4-7 June 2018.

16. Vilajosana, X.; Tuset, P.; Watteyne, T.; Pister, K. OpenMote: Open-Source Prototyping Platform for the Industrial IoT. In Ad Hoc Networks; Mitton, N., Kantarci, M.E., Gallais, A., Papavassiliou, S., Eds.; Springer International Publishing: Cham, Switzerland, 2015; pp. 211-222.

17. Tuset-Peiró, P.; Vilajosana, X.; Watteyne, T. OpenMote+: A Range-Agile Multi-Radio Mote. In Proceedings of the 2016 International Conference on Embedded Wireless Systems and Networks, EWSN '16, Graz, Austria, 15-17 February 2016; pp. 333-334.

18. Texas Instruments. CC2538 Powerful Wireless Microcontroller System-On-Chip for 2.4-GHz IEEE 802.15.4, 6LoWPAN, and ZigBee ${ }^{\circledR}$ Applications (Rev. SWRS096D); 2015. Available online: http://www.ti.com/lit/ds/ symlink/cc2538.pdf (accessed on 17 September 2019).

19. Atmel. AT86RF215 Device Family: Sub-1GHz/2.4GHz Transceiver and I/Q Radio for IEEE Std 802.15.4-2015 (Rev. 42415E); 2016. Available online: http://ww1.microchip.com/downloads/en/devicedoc/atmel-42415wireless-at86rf215_datasheet.pdf (accessed on 17 September 2019).

(c) 2019 by the authors. Licensee MDPI, Basel, Switzerland. This article is an open access article distributed under the terms and conditions of the Creative Commons Attribution (CC BY) license (http:/ / creativecommons.org/licenses/by/4.0/). 